\title{
Ab initio study of intrinsic profiles of liquid metals and their reflectivity.
}

\author{
B. G. del Rio ${ }^{1, *}$, J. Souto ${ }^{2}$, M. M. G. Alemany ${ }^{2}$, and L. E. González ${ }^{1}$ \\ ${ }^{1}$ Departamento de Física Teórica, Universidad de Valladolid, Valladolid, Spain \\ ${ }^{2}$ Departamento de Física de Partículas, Universidad de Santiago de Compostela, Spain
}

\begin{abstract}
The free surfaces of liquid metals are known to exhibit a stratified profile that, in favourable cases, shows up in experiments as a peak in the ratio between the reflectivity function and that of an ideal step-like profile. This peak is located at a wave-vector related to the distance between the layers of the profile. In fact the surface roughness produced by thermally induced capillary waves causes a depletion of the previous so called intrinsic reflectivity by a damping factor that may hinder the observation of the peak. The behaviour of the intrinsic reflectivity below the layering peak is however far from being universal, with systems as $\mathrm{Ga}$ or In where the reflectiviy falls uniformly towards the $q \rightarrow 0$ value, others like $\mathrm{Sn}$ or Bi where a shoulder appears at intermediate wavevectors, and others like $\mathrm{Hg}$ which show a minimum. We have performed extensive ab initio simulations of the free liquid surfaces of $\mathrm{Bi}, \mathrm{Pb}$ and $\mathrm{Hg}$, that yield direct information on the structure of the profiles and found that the macroscopic capillary wave theory usually employed in order to remove the capillary wave components fails badly in some cases for the typical sample sizes affordable in ab initio simulations. However, a microscopic method for the determination of the intrinsic profile is shown to be succesful in obtaining meaningful intrinsic profiles and corresponding reflectivities which reproduce correctly the qualitative behaviour observed experimentally.
\end{abstract}

\section{Introduction.}

Since the original prediction of Rice and coworkers that free liquid surfaces (FLS) of metals would exhibit surface layering in the density profile (DP) perpendicular to the interface [1], $\rho(z)$, almost a decade had to ellapse until this expectation was realized experimentally for the case of liquid $\mathrm{Hg}[2,3]$, through the analysis of the x-ray reflectivity of the FLS, $R\left(q_{z}\right)$, which showed a peak related to the layering. Since then, up to 2009 [4], measurements have been performed for other five pure liquid metals, namely Ga, In, K, Sn and Bi [5]. All these studies were possible due to the advent of $\mathrm{x}$-ray sources intense enough and reflectometers precise enough to measure reflectivities that decay by as much as $9 / 10$ orders of magnitude as the wavevector increases above the position of the reflectivity peak. The experimental

\footnotetext{
*e-mail: beatriz@metodos.fam.cie.uva.es
} 
requirements to make such experiments feasible, as discussed in [5], basically limit the set of pure liquid metals candidates to these studies to those already mentioned, plus liquid $\mathrm{Pb}$ and possibly liquid Ge. A particular source of complications due to the liquid nature of the samples is the presence of thermally induced capillary waves $(\mathrm{CW})$ which blur the so-called intrinsic profile, $\rho_{\text {int }}(z)$, leading to an average profile, $\rho_{\text {av }}(z)$, and deplete even more the measured reflectivity, $R_{m}\left(q_{z}\right)$. The effects of $\mathrm{CW}$ are removed from $R_{m}\left(q_{z}\right)$ through the use of the macroscopic CW theory, that boils down to a division of $R_{m}\left(q_{z}\right)$ by a gaussian, Debye-Waller-like, factor that depends on a capillary roughness, $\sigma_{\mathrm{cw}}$, leading to the intrinsic reflectivity, $R_{\mathrm{int}}\left(q_{z}\right)$. In most cases, this is further divided by the Fresnel reflectivity, $R_{F}\left(q_{z}\right)$, that corresponds to a sharp flat surface and accounts for the overall decay of $R_{\text {int }}\left(q_{z}\right)$. The resulting magnitude is called the surface structure factor, $\left|\Phi_{\text {int }}\left(q_{z}\right)\right|^{2}$, which is directly related to the Fourier Transform of the electronic DP. Note that the capillary roughness depends on the temperature, $T$, and the surface tension, $\gamma$, of the liquid, on the atomic size, and on the lateral resolution of the detector.

The systems studied can be classified in three groups so far: those whose $\left|\Phi_{\text {int }}\left(q_{z}\right)\right|^{2}$ decays monotonously from the layering peak towards $q_{z} \rightarrow 0$ ( $\mathrm{Ga}$, In and $\mathrm{K}$ ), those that display a shoulder at an intermediate $q_{z}(\mathrm{Sn}$ and $\mathrm{Bi}$ ) and those that display a minimum at an intermediate $q_{z}(\mathrm{Hg})$. The origin of such different behaviors must lie in the different electronic DPs, but these are not directly available to experiments. Instead model profiles are proposed and the corresponding reflectivities are fitted to the measurements. The systems in the first group can be adequately described by a simple model named distorted crystal model (DCM), in which the DP is described by layers of equally dense and equispaced gaussians of linearly increasing variance as the bulk is approached. The other groups require some modifications to this model, changing either the positions or the density of some layer(s), in particular the outermost one. However the lack of uniqueness of model profiles that reproduce a given reflectivity poses a note to take into account in the interpretation.

An alternative route to study the FLS of metals is the use of computer simulations. These, however, rely on the availability of methods to describe properly the interaction among ions immersed in a valence electron sea, as adequate for a metal. In this respect, ab initio methods are certainly advantageous, since the underlying density functional theory (DFT) [6] shows a high degree of accuracy. Moreover, simulations give access to the atomic positions, so the DPs can be directly computed, and through them, the reflectivity. In fact, ab initio simulations have already been performed for the FLS of $\mathrm{Si}[7], \mathrm{Na}[8], \mathrm{Sn}[9], \mathrm{Hg}[10]$ and $\mathrm{Cd}[11]$.

The sample size in simulations is utterly smaller than in experiments and consequently the abundance of $\mathrm{CW}$ is much reduced, but it is not null. It is therefore important to take into account this difference for proper comparison with experimental data. In previous studies the procedure adopted has been the same macroscopic $\mathrm{CW}$ theory as used in experiments, except for the aforementioned size differences. However a more direct access to the intrinsic profile and reflectivity is desirable, considering that the simulation samples are very far from being macroscopic. One such method was proposed by Chacon and Tarazona (CT) [12]. It relies on the calculation of a surface that separates the liquid and vapour phases, and sample the atomic positions with respect to this surface in order to obtain the intrinsic profile. The surface is computed so as to be the one that goes through the "surface atoms" and has "minimum area". Further methods have also been proposed, as the so called instaneous liquid-vapor interface [13], which has been used for the analysis of molecular dynamics simulations of the free surface of liquid water [14], but in this contribution we stick 
Table 1. Simulation details.

\begin{tabular}{ccccccc}
\hline & $\rho_{i}\left(\AA^{-3}\right)$ & $T(\mathrm{~K})$ & $N$ & $L_{x}=L_{y}(\AA)$ & $L_{z}(\AA)$ & $N_{c}$ \\
\hline $\mathrm{Hg}$ & 0.0403 & 300 & 116 & 13.03 & 17.10 & 8000 \\
$\mathrm{Bi}$ & 0.0288 & 800 & 160 & 13.57 & 38.69 & 27000 \\
$\mathrm{~Pb}$ & 0.0309 & 850 & 160 & 13.57 & 37.33 & 27000 \\
\hline
\end{tabular}

to the CT procedure, which has already proved fruitful in the analysis of the FLS of molten potasium [15].

In this contribution we report a reanalysis of previous ab initio molecular dynamics (MD) simulations of the FLS of $\mathrm{Hg}$, as well as new calculations for $\mathrm{Pb}$ and $\mathrm{Bi}$. We discuss the differences between the average and intrinsic profiles as obtained through the $\mathrm{CT}$ procedure, highlighting the advantages of the latter. Also, when available, we compare our reflectivities with the measured ones.

\section{Theory.}

The energy and the forces on the atoms for each MD configuration were computed through the use of DFT and pseudopotentials to describe the interaction between ions and valence electrons. The simulations for $\mathrm{Hg}[10]$ were performed using the Quantum Espresso (QE) code [16], and an ultrasoft pseudopotential with 12 valence electrons. In the cases of $\mathrm{Pb}$ and $\mathrm{Bi}$ we used the PARSEC code [17] along with norm-conserving pseudopotentials with 4 and 5 valence electrons respectively [18, 19]. The PARSEC code is very efficient, but it is not suited for ultrasoft pseudopotentials, which are a must for liquid $\mathrm{Hg}$.

For the study of the FLSs we used $N$ atoms in a slab geometry of initial width adequate to the experimental density, $\rho_{i}$, located in a tetragonal simulation box with $L_{z}>L_{x}=L_{y}$ and periodic boundary conditions. The vacuum region separating the replicas of each slab is taken large enough so that the inter-slab interaction can be safely neglected. After equilibration at the desired temperature $N_{c}$ microcanonical configurations were generated from which the different properties were sampled. The particular values used for the different systems are shown in table 1 .

The average electronic DP is obtained from the evaluation of a histogram of the $z$-coordinates of the particles, after representing each particle by the projection of the atomic electron density onto the $z$-direction.

The CT procedure to obtain the intrisic DP for a FLS relies on the computation, for each MD configuration, of a liquid-vapour separation surface, $z=\xi(x, y)$, so that the intrinsic DP is obtained from a histogram of the particles distances to this surface along the $z$-direction, i.e., according to their values of $z_{i \text {;rel }}=z_{i}-\xi\left(x_{i}, y_{i}\right)$, where $i$ runs over all the particles of the sample. The separation surface must comply with the periodic boundary conditions (PBC) of the simulation cell, so it is represented by its Fourier series. The separation surface should not oscillate within an atomic diameter. Therefore the series is terminated at $q$-values correponding to this size and compatible with the $\mathrm{PBC}$, and consequently has a finite number of coefficients. These coefficients are determined by a constrained minimization of the surface area. The constraints imposed consist in the requirement that the separation surface passes through the surface atoms (pivots), i.e., $z_{\ell}=\xi\left(x_{\ell}, y_{\ell}\right)$, with $\ell$ running through the pivots. For each configuration 9 atoms are initially selected as pivots, by taking those whose $z$-coordinate is smallest among the atoms in each of the $3 \times 3$ prisms obtained 
by partitioning the simulation cell equally across the $x y$ plane. After computing the minimal surface that passes through these pivots we locate the atom closest to this surface and include it as a new pivot. Further recalculations of minimal surfaces that pass through the pivots and inclusions of the closest atom as pivot are repeated until a specified number of pivots is reached, which is 21 atoms for $\mathrm{Hg}$ and 19 atoms for $\mathrm{Pb}$ and $\mathrm{Bi}$. This number is self-consistently obtained from the method (and of course depends on the sample sizes), because the use of a different number of final pivots leads to intrinsic profiles with strange shapes that include shoulders at either the outermost layer or the first inner one. After the Fourier coefficients have been obtained one can optionally truncate the series, while keeping the coefficients, at lower cutoff wavevectors. This leads to a smoother separation surface which does not go exactly trough all the pivots, but close to them. Such separation surface is more physically appealing, since in the liquid there are not only capillary waves, but also other types of excitations that influence the vertical motion of the surface atoms, for instance transverse waves that propagate parallel to the surface. Due to the limited size of the samples used in ab initio simulations this further truncation of the series can only be performed one level, down to the next lower $q$ compatible with the PBC.

Finally, after the DPs have been computed the reflectivities are obtained from the relations

$$
\Phi\left(q_{z}\right)=\int_{0}^{\infty} d z \exp \left[-i q_{z} z\right] \frac{d \rho(z) / \rho_{0}}{d z} \quad \frac{R\left(q_{z}\right)}{R_{F}\left(q_{z}\right)}=\left|\Phi\left(q_{z}\right)\right|^{2} .
$$

where $\rho_{0}$ is the density of the bulk liquid. When $\rho(z)$ is the intrinsic profile these relations lead to the intrinsic reflectivity. However, when $\rho(z)$ is the average profile the resulting reflectivity function includes the $\mathrm{CW}$ present in the simulation. From this the corresponding intrinsic reflectivity, within the macroscopic $\mathrm{CW}$ theory, is obtained through the relation

$$
\frac{R_{\mathrm{int}}^{\mathrm{CWT}}\left(q_{z}\right)}{R_{F}\left(q_{z}\right.}=\frac{R_{\mathrm{av}}\left(q_{z}\right) / R_{F}\left(q_{z}\right)}{\exp \left[-\sigma^{2} q_{z}^{2}\right]}
$$

where $\sigma^{2}$ includes the capillary roughness and also an intrinsic roughness, not really well defined but related to the atomic size [10].

\section{Results and discussion.}

\subsection{Liquid $\mathrm{Hg}$}

The experiments on liquid $\mathrm{Hg}$ at several temperatures [3], without correcting for $\mathrm{CW}$, yielded a $R_{m}\left(q_{z}\right) / R_{F}\left(q_{z}\right)$ with a layering peak at $q_{z}=2.2 \AA^{-1}$ and a minimum at intermediate $q$ between 0.6 and $1.2 \AA^{-1}$ depending on the sample measured. A first attempt to fit the data with the DCM lead to an interlayer spacing of $\lambda=2.72 \AA$, but the fit was not good. Two modifications were proposed. The first one included a quite broad ad-layer of very small density $\left(0.1\right.$ to $\left.0.3 \rho_{0}\right)$ located a few $\AA$ into the vapour region. The second one corresponded to an interface layer broadened and a little depleted and with an expanded distance to the next layer. This second model was more in line with the one used in the first measurements [2], for which the first interlayer distance was $\lambda_{1}=3.05 \pm 0.15 \AA$, and the rest were $\lambda=2.76 \pm 0.20 \AA$. The surface roughness was estimated as $\sigma \approx 1.0 \AA$.

Our simulations [10] led to an average profile, shown in fig. 1 , with $\lambda_{1} \approx 2.95$ and $\lambda \approx 2.75$, in qualitative agreement with experiment. Moreover, the reflectivity, 


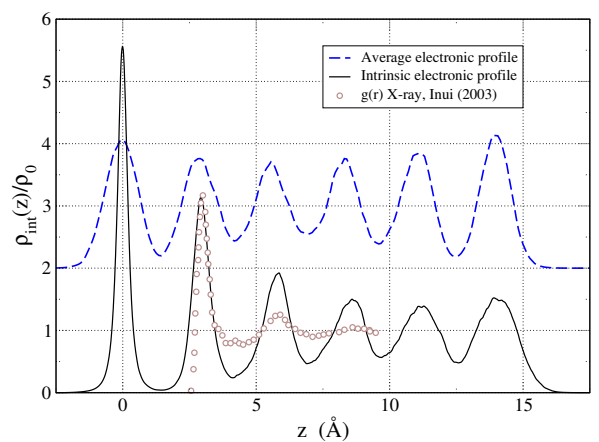

Figure 1. Intrinsic and average electronic density profiles for $\mathrm{Hg}$ at $300 \mathrm{~K}$. Also shown is the experimental pair correlation function of the bulk liquid.

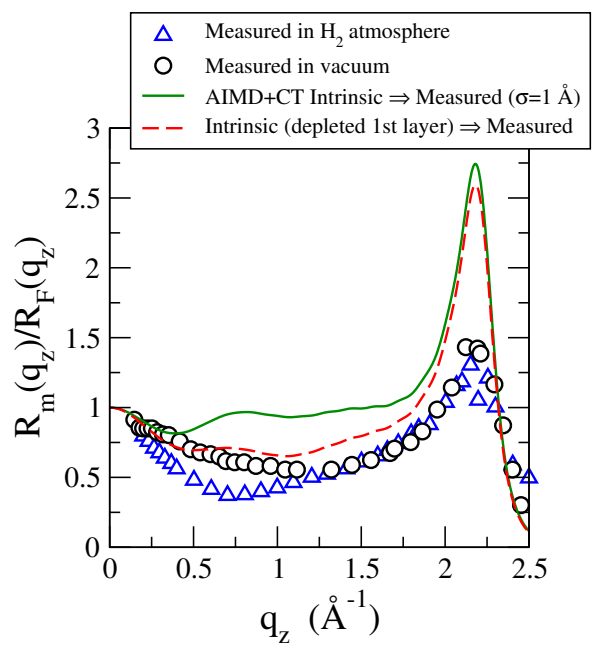

Figure 2. Reflectivities for $\mathrm{Hg}$ at $300 \mathrm{~K}$.

modified through the CWT to be directly comparable to the measured one, showed a peak at a similar $q_{z}$ as the experiment but did not reproduce the intermediate minimum. The intrinsic electronic profile computed through the CT procedure is also shown in fig. 1 and leads to the same $\lambda_{1}$ as the average profile, whereas the inner interlayer distance increases a little to $\lambda \approx 2.84 \AA$. The layers are however narrower and higher than in $\rho_{\text {av }}(z)$. The layer positions correlate very well with the available pair distribution function of the bulk liquid [20]. It is interesting to note that $\rho_{\text {int }}(z)$ cannot be fit by the models used by experimentalists, since the first two layers don't have a gaussian shape, although they can be reproduced as the sum of two gaussians for each layer. The resulting intrinsic reflectivity has been modified through the same procedure and using the same parameters as in experiment [3] and is shown in fig. 2 . It keeps the correct peak position, although the height is overestimated, but it still does not produce the dip found in the measurements. Nevertheless, the form of the reflectivity is very sensitive to small details in the profile, and a $10 \%$ reduction of the height of the first layer does produce a correct minimum in the $q_{z}$ region where it was experimentally observed. Therefore we can conclude that the expanded distance and a small depletion of the fist layer are behind the appearance of the minimum, in line with the second experimental model.

\subsection{Liquid $\mathrm{Pb}$ and $\mathrm{Bi}$}

In table 1 one can observe a much higher number of configurations for these two systems than those for $\mathrm{Hg}$. The main reason was the lack of convergence of the average DP as obtained directly from the simulations. We observed that further increase in $N_{c}$ did not lead to any substantial improvement in the convergence. We performed several tests, using different simulation cells, different pseudopotentials (ultrasoft vs norm-conserving) and/or different computer codes (QE and VASP vs PARSEC), but the lack of convergence persisted in all cases. We consider that such problems in the simulation, not present in $\mathrm{Hg}$, are due to the combination of a high temperature and low surface tension, both favouring large fluctuations in the height 


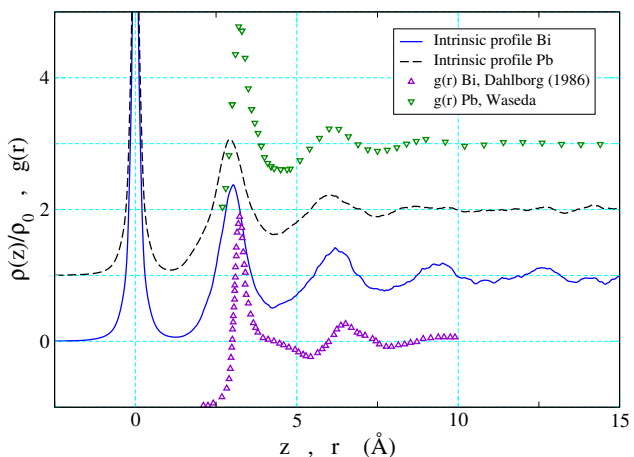

Figure 3. Intrinsic electronic density profiles for $\mathrm{Bi}$ and $\mathrm{Pb}$. Also shown are the experimental pair correlation functions of the bulk liquids. The different functions are shifted vertically for clarity.

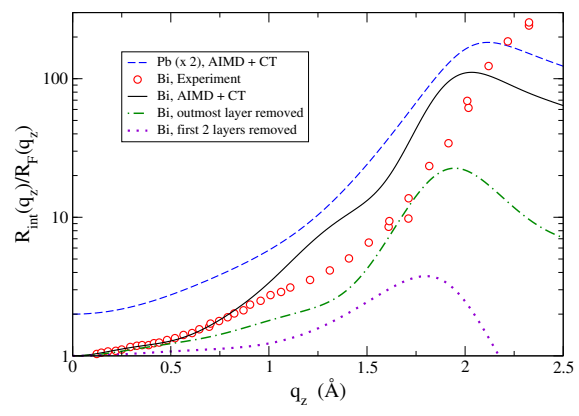

Figure 4. Reflectivities for liquid $\mathrm{Bi}$ and $\mathrm{Pb}$ (multiplied by two for clarity).

of the surface atoms, which are enhanced even more by the small number of them at the surface layer.

No experiments have been performed for the FLS of $\mathrm{Pb}$. On the contrary, $\mathrm{Bi}$ (together with $\mathrm{Sn}$ ) is one of the systems whose measured reflectivity, when transformed into the intrinsic one, shows a shoulder at intermediate $q$ values [4] between 0.6 and 1.4 $\AA^{-1}$. The authors constructed several models that could fit the measured reflectivity, with an adlayer on top of a DCM. The main conclusion was that the adlayer should be denser than the bulk in order to produce the shoulder, but different values of the width, position and density of the adlayer could do the job. The inner interlayer distance, as obtained from the DCM, was $\lambda \approx 2.83 \pm 0.05 \AA$.

The intrinsic electronic $\mathrm{DP}$ obtained for $\mathrm{Bi}$ and $\mathrm{Pb}$ are shown in fig. 3, along with the corresponding experimental $g(r)[21,22]$. The shape of the layers is not DCM-like, requiring several gaussians for a proper fit. The layer positions in general agree with the maxima of $g(r)$ although some relaxation towards smaller distances is observed in the profile. Moreover, specific features as the shoulder of $g(r)$ in $\mathrm{Bi}$, are reflected also in the shape of $\rho_{\text {int }}(z)$. The inner interlayer distance in $\mathrm{Bi}$ is found to be $\approx 3.12 \AA$, somewhat larger than the one experimentally determined through the fit to $R_{m}\left(q_{z}\right)$. Despite the many non-DCM features of the profile, its form is in line with one of the possible models put forward by Pershan and coworkers, with a very narrow outmost layer, at a slightly contracted distance to the inner layer $\left(\lambda_{1}=3.02 \AA\right)$ and with a slightly enhanced integrated density.

The corresponding intrinsic reflectivities are displayed in fig. 4 along with the experimental one for $\mathrm{Bi}$ [4]. In $\mathrm{Pb}$ we find a monotonous function, akin to those of $\mathrm{Ga}$, In and $\mathrm{K}$. For $\mathrm{Bi}$ we find, in agreement with experiments, a shoulder at intermediate $q_{z}$, although the magnitude of the reflectivity is rather overshot. To check the experimentalists suggestion that an enhanced first layer is responsible for the shoulder, we decreased the density of this layer, obtaining still a reflectivity with a shoulder. However, including one by one layers from the bulk towards the surface for computing $R_{\text {int }}\left(q_{z}\right)$ we find that the shouldered structure appears after the first inner layer is 
included. Consequently we can conclude that the two first layers are responsible for this kind of behaviour.

\section{Conclusions.}

The first important point to note is that the CT procedure leads to converged DPs, which is not always the case for the average profile, possibly due to a combination of high temperature and low surface tension.

The combination of AIMD simulations and the CT procedure leads to reflectivities which, either directly or with small modifications, agree qualitatively with experiments.

The profiles obtained are not in general well described by the DCM or variations thereof, but the general characteristics of the intrinsic profiles are similar to the models proposed by the experimentalists. We observe a high degree of correlation between the bulk $g(r)$ and the characteristics of the intrinsic profile, although with some relaxation in the distances, which become somewhat smaller. The particular behaviour of the reflectivities has been traced back to the depletion of the first layer in the case of $\mathrm{Hg}$, and to a combined effect of the first two layers in the case of $\mathrm{Bi}$.

We acknowledge the support of MECD (FIS2012-33126 and FIS2014-59279-P), Xunta de Galicia (AEFIS: AGRUP2015/11) and JCyL (VA104A11-2). BGR acknowledges the financial support of the Universidad de Valladolid.

\section{References}

[1] M. P. d'Evelyn and S. A. Rice, Phys. Rev. Lett. 47, 1844 (1981).

[2] O. M. Magnussen et al, Phys. Rev. Lett. 74, 4444 (1995).

[3] E. DiMasi et al, Phys. Rev. B 58, R13419 (1998).

[4] P. S. Pershan et al, Phys. Rev. B 79, 115417 (2009).

[5] P. S. Pershan and M. L. Schlossman, Liquid Surfaces and Interfaces. Synchrotron X-ray Methods, (New York: Cambridge, 2012), and references therein.

[6] P. Hohenberg and W. Kohn, Phys. Rev. 136, B864 (1964); W. Kohn and L.J. Sham, Phys. Rev. 140, A1133 (1965).

[7] G. Fabricius et al, Phys. Rev. B 60, R16283 (1999).

[8] B. G. Walker, C. Molteni and N. Marzari, J. Phys.: Condens. Matter 26, S2575 (2004).

[9] L. Calderín, L. E. González and D. J. González, Phys. Rev. B 80, 115403 (2009).

[10] L. Calderín, L. E. González and D. J. González, Phys. Rev. B 87, 014201 (2013).

[11] L. Calderín, L. E. González and D. J. González, J. Phys.: Condens. Matter 25, 065102 (2013).

[12] E. Chacón and P. Tarazona, Phys. Rev. Lett. 91, 166103 (2003).

[13] A. P. Willard and D. Chandler, J. Phys. Chem. B 114, 1954 (2010).

[14] J. Kessler et al, J. Phys. Chem. B 119, 10079 (2015)

[15] E. Chacón, P. Tarazona and L. E. González, Phys. Rev. B 74, 224201 (2006).

[16] P. Gianozzi et al, J. Phys.: Condens. Matter 21, 395502 (2009).

[17] M. M. G. Alemany et al, Phys. Rev. B 69, 075101 (2004); L. Kronik et al, Phys. Status Solidi B 243, 1063 (2006); M. M. G. Alemany et al, Comput. Phys. Comm. 177, 339 (2007).

[18] M. M. G. Alemany et al, Phys. Rev. B 76, 214203 (2007). 
[19] J. Souto et al, Phys. Rev. B 81, 134201 (2010).

[20] M. Inui, X. Hong and K. Tamura, Phys. Rev. B 68, 094108 (2003).

[21] U. Dahlborg and M. Davidovič, Phys. Chem. Liq. 15, 243 (1986).

[22] Y. Waseda, http://res.tagen.tohoku.ac.jp/ waseda/scm/LIQ/all/pb.html 